\title{
Evaluation of the anisotropic radiative conductivity of a low-density carbon fiber material from realistic microscale imaging
}

\author{
Nima Nouri ${ }^{\mathrm{a}, \mathrm{c}}$, Francesco Panerai ${ }^{\mathrm{a}}$, Kaveh A. Tagavi ${ }^{\mathrm{a}}$, Nagi N. Mansour ${ }^{\mathrm{d}}$, Alexandre Martin ${ }^{\mathrm{a}, \mathrm{b}, *}$ \\ ${ }^{a}$ Department of Mechanical Engineering, University of Kentucky, Lexington, KY 40506, USA \\ ${ }^{b}$ Center for Computational Science, University of Kentucky, Lexington, KY 40506, USA \\ ${ }^{c}$ Department of Physics and Astronomy, University of Kentucky, Lexington, KY 40506, USA \\ ${ }^{d}$ NASA Advanced Supercomputing (NAS) Division, NASA Ames Research Center, Moffett Field, CA, 94035, USA
}

\begin{abstract}
The radiative heat transfer inside a low-density carbon fiber insulator is analyzed using a three-dimensional direct simulation model. A robust procedure is presented for the numerical calculation of the geometric configuration factor to compute the radiative energy exchange processes among the small discretized surface areas of the fibrous material. The methodology is applied to a polygonal mesh of a fibrous insulator obtained from three-dimensional microscale imaging of the real material. The anisotropic values of the radiative conductivity are calculated for that geometry. The results yield both directional and thermal dependence of the radiative conductivity.
\end{abstract}

Keywords: Radiative heat transfer, Porous media, Radiative conductivity, Effective conductivity, Thermal Protection Systems

\section{Introduction}

For highly porous media subjected to high temperature and high temperature gradients, radiative energy transport within the material is dominant. This radiative heat transfer can be expressed in the form of a radiative conductivity [1]. In such a model, the thermal conductivity is highly dependent on the temperature. An example of an application where this type of heat transfer is important is in evaluating the design of Thermal Protection Systems (TPS) for planetary probes and space vehicles $[2,3,4]$.

In a previous study [5], a three-dimensional direct simulation model was developed to calculate the radiative conductivity of fibrous material using a simplified geometric model with properties resembling those of a carbon fiber insulator. It was shown that the radiative conductivity was a function of the temperature and the

\footnotetext{
${ }^{*}$ Corresponding author at: Department of Mechanical Engineering, University of Kentucky, Lexington, KY 40506, USA. Tel.: +1 (859) 2574462.

Email addresses: Nima. Nouri@uky . edu (Nima Nouri),

Francesco.Panerai@uky.edu (Francesco Panerai),

Kaveh.Tagavi@uky . edu (Kaveh A. Tagavi),

Nagi.N.Mansour@nasa.gov (Nagi N. Mansour),

Alexandre.Martin@uky.edu (Alexandre Martin)
}

orientation of the fibers because of the anisotropic nature of the test geometry.

In the present paper, a three-dimensional model obtained from X-ray microtomography $[6,7]$ is used so that the geometric properties of the fibrous material at the microscopic scale can be better captured as manufactured. More specifically, a rendering of FiberForm ${ }^{\circledR}$ is utilized. FiberForm ${ }^{\circledR}$ is a carbon fiber insulator that serves as the preform to certain TPS materials [8]. To demonstrate the validity of the approach documented in this paper, the effective conductivity tensor of the sample is calculated, and compared to the experimental results.

\section{Geometric configuration factor calculation and analysis}

The calculation of the geometric configuration factor (GCF) is an important step when determining the radiative exchange between two surface elements (or "surfaces"). The computation of GCF involves integrating over the solid angles subtended by each surface element, if they are in sight to each other. In the absence of an exact analytical solution for the GCF, numerical integration is required. Since complex geometries require a discretized geometry with many small surface

September 29, 2015 


\begin{tabular}{|c|c|c|}
\hline \multicolumn{3}{|l|}{ Nomenclature } \\
\hline $\begin{array}{ll}\text { Symbols } & \\
A & \text { area of an internal enclosure }\left[\mathrm{m}^{2}\right] \\
a & \text { area of a triangular surface element }\left[\mathrm{m}^{2}\right] \\
C & \text { coordinate of the centroid }[\mathrm{m}] \\
F & \text { geometric configuration factor }(\mathrm{GCF}) \\
K & \text { geometric factor [m] } \\
L & \text { length of the enclosure [m] } \\
N & \text { number of surfaces within enclosure } \\
q & \left.\text { heat flux [W } \mathrm{W}^{-2}\right] \\
S & \text { distance between two surface elements [m] } \\
T & \text { temperature }[\mathrm{K}] \\
x, y, z & \text { spatial Cartesian coordinates }[\mathrm{m}] \\
\text { Greek Symbols } \\
\kappa & \left.\text { radiative conductivity [W } \mathrm{m}^{-1} \mathrm{~K}^{-1}\right] \\
\Lambda & \text { principal-axis geometric factor }[\mathrm{m}] \\
\lambda & \text { principal-axis radiative conductivity }[\mathrm{W} \\
& \left.\mathrm{m}^{-1} \mathrm{~K}^{-1}\right]\end{array}$ & $\begin{array}{l}\sigma \\
\theta \\
\varepsilon \\
\zeta \\
\text { Superscril } \\
\text { eff } \\
\text { sol } \\
\text { Subscripts } \\
\ell, m, n \\
I, I I \\
i, j, k \\
\text { IP } \\
T T T \\
\text { irr } \\
\text { rds } \\
\text { fin } \\
\text { ini }\end{array}$ & $\begin{array}{l}\text { Stefan-Boltzmann constant }\left[\mathrm{W} \mathrm{m}^{-2} \mathrm{~K}^{-4}\right] \\
\text { angle between the normal and } S \\
\text { emissivity } \\
\text { error due to grid resolution } \\
\text { pts } \\
\text { effective } \\
\text { solide } \\
\text { directional indices } \\
\text { opposite side enclosure walls indices } \\
\text { surface index } \\
\text { In-plane } \\
\text { through-the-thickness } \\
\text { irradiation } \\
\text { radiosity } \\
\text { final } \\
\text { initial }\end{array}$ \\
\hline
\end{tabular}

elements to preserve accuracy, a robust procedure is required. Theoretical details and general expressions of the GCF numerical calculation were presented and verified in a previous study [5].

The numerical rendering of the surface of a complex geometry is often performed using a mesh of flat, triangular elements. In the limit of transparent gas, the energy exchange between the individual elements, considered to be isothermal, opaque, diffuse and gray, contributes to the radiative energy exchange.

To ensure that all radiative contributions are properly accounted for, the geometry is enclosed in a cubic fiducial volume (see Fig. 1(a)). Each boundary wall, called internal enclosure (IE), is composed of the same material as the fibrous material (isothermal, opaque, diffuse and gray).

\subsection{Surface-to-surface GCF calculation}

The GCF is calculated for three different cases: (1) triangle-to-triangle, (2) IE-to-triangle and (3) IE-to-IE. The expression corresponding to each case is listed in Table 1 , where $\theta_{i}$ is the angle between the normal of the surface elements and the line of length $S_{i j}$ joining the centroid of the triangles $i$ and $j$, of area $a_{i}$ and $a_{j}$, respectively. Each IE surface is divided into a set of uniform subsurfaces $\{\Delta A\}$ (see Fig. 1(b)) to perform the discretized integration. The size of $\Delta A$ is taken to be

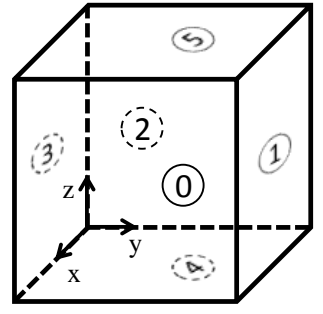

(a) Cubic enclosure containing the complex geometry, with internal enclosure (IE) surfaces identified by numbers

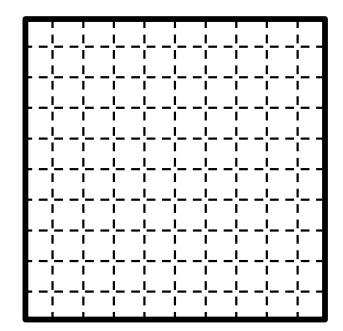

(b) Square mesh of subsurface $\Delta A$ superposed on an internal enclosure (IE) surface of area $A$ so that $\sum \Delta A=$ A.
Figure 1: Schematic figure of the specific experimental geometry employed in the model.

small enough so that the results are considered mesh independent, with a relative error of less than $10^{-9}$. The reciprocal relations corresponding to each case is also listed in Table 1.

\subsection{Acceleration of the GCF calculation}

For $N$ surfaces, owing to the reciprocity relation, only $N(N-1) / 2$ GCFs need to be calculated by integration. The calculation can be further accelerated by taking advantage of the geometric properties of the surface: 
Table 1: Geometric configuration factor and reciprocity relations used in the model. Case (1) Triangle $i$ to triangle $j$. Case (2) Subsurface $k$ of IE $j$ to triangle $i$. Case (3) Subsurface $k$ of IE $i$ to subsurface $\ell$ of IE $j$.

\begin{tabular}{lll}
\hline Case & Geometric configuration factor & Reciprocity \\
\hline 1 & $F_{i \rightarrow j}=a_{j} \frac{\cos \theta_{i} \cos \theta_{j}}{\pi S_{i j}^{2}}$ & $a_{i} F_{i \rightarrow j}=a_{j} F_{j \rightarrow i}$ \\
2 & $F_{i \rightarrow j}=\frac{\Delta A}{\pi} \sum_{k} \frac{\cos \theta_{i} \cos \theta_{k}}{S_{i k}^{2}}$ & $a_{i} F_{i \rightarrow j}=A_{j} F_{j \rightarrow i}$ \\
3 & $F_{i \rightarrow j}=\frac{(\Delta A)^{2}}{\pi A_{i}} \sum_{k, \ell} \frac{\cos \theta_{k} \cos \theta_{\ell}}{S_{k \ell}^{2}}$ & $A_{i} F_{i \rightarrow j}=A_{j} F_{j \rightarrow i}$ \\
\hline
\end{tabular}

Field-of-view. A non-zero GCF exists if and only if surfaces $i$ and $j$ are in sight to each other. Defining $\theta$ as the angle between the normal of the surface element and the line joining the centroid of two triangles, then $F_{i \rightarrow j}=F_{j \rightarrow i}=0$ if $\theta \notin[0, \pi / 2[$.

Obstruction. In case of isotropic emission (diffuse-gray approximation), radiation exchange between two surfaces is possible if and only if their line of sight is not obstructed by an opaque object. This occurs when an opaque surface intersects the straight path from centroid $i$ to centroid $j$. If this condition is met, then $F_{i \rightarrow j}=F_{j \rightarrow i}=0$.

It is worth noting that these two procedures do not necessarily guarantee that the radiation is completely blocked. In some cases based on the size and orientation of the triangles, the energy can be partially exchanged, since portions of surfaces are still in sight of each other. This inaccuracy may be mitigated by using a fine mesh of good quality [9], with triangles of approximately uniform area.

\subsection{Refinement of the GCF calculation}

As the GCF is calculated, two additional procedures are performed to ensure that the discretization of the domain is sufficiently refined:

Bounding. If the calculated $F_{i \rightarrow j}$ is greater than 1 , then the mesh is considered to be too coarse. In the first equation of Table 1 , since $\cos \theta_{i} \cos \theta_{j}$ is always $\in[0,1]$, it is possible to ensure that the mesh is sufficiently refined by requiring that $a_{j} /\left(\pi S_{i j}^{2}\right) \leq 1$ over all triangles.

Closure. In an enclosure, the sum of all view factors $F_{i \rightarrow j}$ from a given surface $i$ must equal to 1 (i.e. $\left.\sum_{j} F_{i \rightarrow j}=1\right)$. Due to the numerical errors associated with the discretized surface elements, the summation is usually smaller than 1 (i.e. $\sum_{j} F_{i \rightarrow j}=1-\xi_{i}$ where $\xi_{i} \sim 10^{-3}$ ). To remediate this, the value of $\xi_{i}$ is distributed among all configuration factors of $i$ such that the summation becomes 1 .

\section{Radiation exchange and effective heat conduction model}

In a black-body radiation approximation, where absorptivity and emissivity $(\varepsilon)$ coefficients are equal, the balance equation between radiosity $q_{\mathrm{rds}}$ and irradiation $q_{\text {irr }}$ at temperature $T$ for the $i^{\text {th }}$ surface element is given by [10]:

$$
q_{\mathrm{rds}, i}=\varepsilon \sigma T_{i}^{4}+(1-\varepsilon) q_{\mathrm{irr}, i},
$$

where $\sigma=5.67 \times 10^{-8} \mathrm{~W} /\left(\mathrm{m}^{2} \cdot \mathrm{K}^{4}\right)$ is the Stefan-

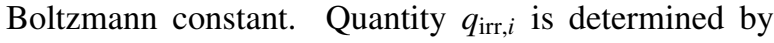
summing over the portions of radiation within the fiducial volume that reaches surface $i$. Using the reciprocity relation, Eq. (1) becomes:

$$
q_{\mathrm{rds}, i}=\varepsilon \sigma T_{i}^{4}+(1-\varepsilon) \sum_{j} F_{i \rightarrow j} q_{\mathrm{rds}, j} .
$$

For the IE, a re-radiating boundary condition is applied such that $q_{\text {rds }}=q_{\text {irr }}$, which can be written as the following:

$$
q_{\mathrm{rds}, i}=\sum_{j} F_{i \rightarrow j} q_{\mathrm{rds}, j}
$$

Equations (2) and (3) form a $N \times N$ system of equations, which can be solved to obtain the values of radiosities $\left\{q_{\mathrm{rds}, i}\right\}$.

In order to determine the effective conductivity of a volume due to radiative transfer, the energy flux passing through the fiducial volume is modeled assuming a heat conductivity type relation. As illustrated in Fig. 3, radiative conductivity $\kappa$ is expected to show a directional dependency. Therefore, the law of heat conduction (Fourier's law) for an anisotropic medium is used:

$$
q_{m}=-\sum_{n} \kappa_{m n} \partial_{n} T, \quad m, n \in\{x, y, z\} .
$$

Here, $\partial_{n}$ denotes derivative operator (i.e. $\partial / \partial_{x}, \partial / \partial_{y}$ and $\left.\partial / \partial_{z}\right)$. The $\kappa_{m n}$ elements are determined using a systematic procedure. A linear temperature gradient ranging from $T_{I I}$ to $T_{I}$ is applied along the $n$-direction, such that the $i^{\text {th }}$ triangular surface is assigned temperature:

$$
T_{i}=T_{I I}-\Delta T \frac{C_{n, i}}{L_{n}}
$$


Here, $\Delta T=T_{I I}-T_{I}>0, C_{n, i}$ and $L_{n}$ are $n$-component of $i^{\text {th }}$ triangular centroid coordinate and the distance between the two enclosure walls in the $n$-direction, respectively. Also, a re-radiating boundary condition is imposed for the enclosure walls normal to the $n(m \neq n)$. This temperature gradient is only imposed in the $n-$ direction, therefore leading to $\partial_{m \neq n} T=0$. The planar averaged heat flux vector $q_{m}$ for the imposed temperature is calculated using the difference between the radiosity from one enclosure wall, and the irradiation flux from the opposite wall. Therefore, from Eq. (4), for each $n$-direction, three conductivity elements $\kappa_{m n}$ (where $m=x, y$, and $z$ ) at $T=\left(T_{I}+T_{I I}\right) / 2$ can be determined as:

$$
\kappa_{m n}(T)=-q_{m}(T) \frac{L_{n}}{\Delta T} .
$$

Based on the Rosseland formulation, the radiative conductivity is expected to exhibit a cubic polynomial dependence on temperature [10, Ch. 12]. Therefore, the $\kappa_{m n}$ elements are fitted to a cubic polynomial of temperature,

$$
\kappa_{m n}(T)=K_{m n} \varepsilon \sigma T^{3},
$$

where $K$ is the geometric factor associated with the Rosseland model.

It is important to point out that in this methodology, only the Rosseland temperature-dependency formulation is used, and not the approximate model [11].

\section{Results}

\subsection{Geometry}

The fiducial volume is obtained from a subset of a micro-CT scan of FiberForm ${ }^{\circledR}$, obtained during a previous study $[7,6]$. The fibrous volume is segmented into a surface mesh of 56268 triangular elements, illustrated in Fig. 2. properties of Fig. 2.

\subsection{Geometric configuration factor}

For illustrative purposes, a binned "3D map" of the bilateral GCFs of the geometry is shown in Fig. 3. Each value is obtained by averaging the $56268^{2} F_{i \rightarrow j}$ elements over $100^{2}$ intervals. For instance, $F_{i \rightarrow j}$ values for $(i, j)=(0,0) \rightarrow(561,561)$ are averaged and mapped to the bin $(0,0)$ and the $F_{i j}$ values for $(i, j)=(562,0) \rightarrow$ $(1123,561)$ are averaged and mapped to bin $(1,0)$, and so on. The heterogeneous structure of FiberForm ${ }^{\circledR}$ manifests itself through the non-uniform distribution of the GCFs which consequently leads to a non-uniform energy distribution over all the fiducial volume.

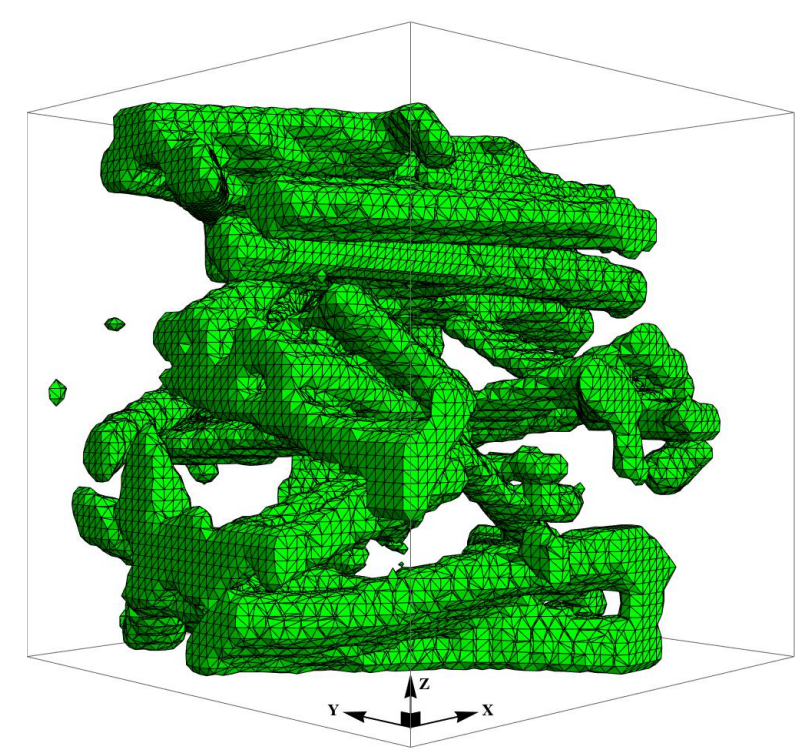

Figure 2: (Color online) Three-dimensional microscopic rendering of FiberForm ${ }^{\circledR}$ extracted from X-ray computed microtomography. The volume is contained in a $0.1 \times 0.1 \times 0.1 \mathrm{~mm}^{3}$ cube, and the fibers have an average diameter of $10.6 \mu \mathrm{m}$.

\subsection{Radiative conductivity}

The analysis for calculating the radiative conductivity (Section 3) is performed for $\Delta T=1.0 \mathrm{~K}$ over the model for a temperature range of $T_{\text {ini }} \leq T \leq T_{\text {fin }}$, where $T_{\text {ini }}=300.0 \mathrm{~K}$ and $T_{\text {fin }}=4000.0 \mathrm{~K}$. For the emissivity, a uniform value of $\varepsilon=0.85$ is used [12]. The calculated temperature-dependent conductivities are shown in Fig. 4. Because the conductivity tensor is known to be symmetric [13], the averaged temperature-dependent values of both $\kappa_{m n}$ and $\kappa_{n m}$ are used to calculate the offdiagonal elements. As expected from the Rosseland model, these results follow the cubic temperature dependency of Eq. 6. Therefore, the geometric factors $K_{m n}$ associated with the best fit obtained from a cubic curve are extracted and presented in Table 2(a). The calculated values illustrate the anisotropic behavior of the selected volume since the diagonal elements are all different.

The radiative conductivity tensor possesses three mutually orthogonal principal axes (i.e. the orientation of the geometry where the off-diagonal terms are zero) which are associated with three real eigenvalues. The Rosseland factor $\Lambda$ for the principal-axis conductivity elements $\lambda$ are listed in Table 2(b) so that

$$
\lambda_{m}(T)=\Lambda_{m} \varepsilon \sigma T^{3} \text {. }
$$




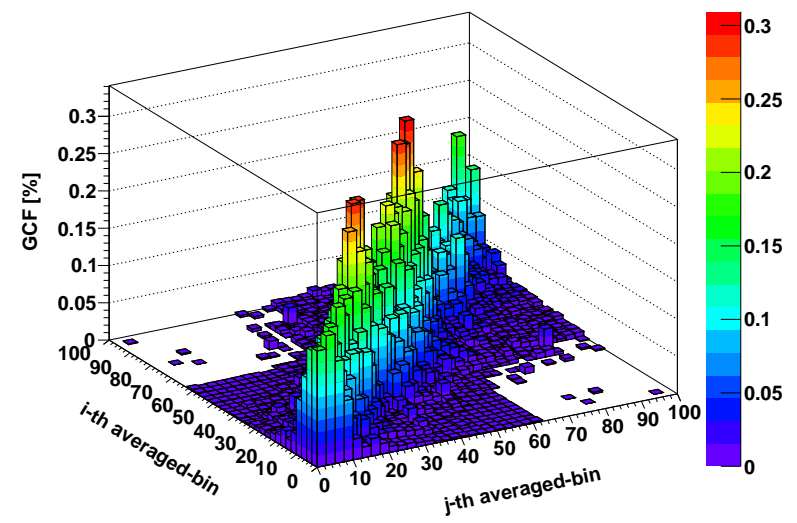

Figure 3: (Color online) Calculated geometric configuration factors have been plotted in a bin-averaged over $562 \times 562$ consecutive $(i, j)$ combinations.

Table 2: (a) Geometric factor reconstructed from Rosseland model, and (b) geometric factor reconstructed from Rosseland model along the principal axis.

\begin{tabular}{|c|c|c|c|}
\hline \multirow[t]{2}{*}{ (a) } & \multicolumn{3}{|c|}{$K\left[10^{-3} \mathrm{~m}\right]$} \\
\hline & $\mathbf{x}$ & $\mathbf{y}$ & $\mathbf{Z}$ \\
\hline $\mathbf{X}$ & 2.072 & 0.207 & 0.130 \\
\hline $\mathbf{y}$ & 0.207 & 1.526 & 0.436 \\
\hline $\mathbf{Z}$ & 0.130 & 0.436 & 1.856 \\
\hline (b) & \multicolumn{3}{|c|}{$\Lambda\left[10^{-3} \mathrm{~m}\right]$} \\
\hline & 1 & 2 & 3 \\
\hline & 2.347 & 1.213 & 1.894 \\
\hline
\end{tabular}

\subsection{Discussion}

The values of the thermal conductivity calculated using the present method can be compared to the values provided by the manufacturer [14], as well as those available in the literature [8]. In these two references, the values in the through-the-thickness (TTT) direction is presented. The TTT direction is defined as $\kappa_{m m} \ll \kappa_{n n}, \kappa_{\ell \ell}$, where $\kappa_{n n} \approx \kappa_{\ell \ell}$ is the in-plane $(I P)$ direction. For FiberForm ${ }^{\circledR}$, these directions are the results of the orientation of the fibers due to the compression axis. Since these results are obtained experimentally, they include all the physical phenomena that contribute to conductivity: the radiative conductivity, the gas phase conductivity (argon), and the solid conductivity. For the radiative conductivity factors calculated in Table 2 , the TTT direction corresponds to the smallest value, the $y-$ axis.

To properly compare the two values, the radiative conductivity calculated in this work is added to the solid

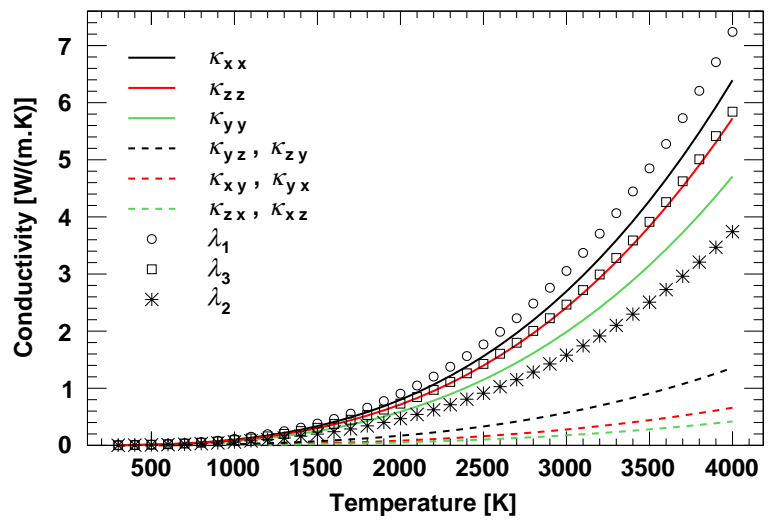

Figure 4: (Color online) Radiative conductivity matrix elements as a function of temperature.

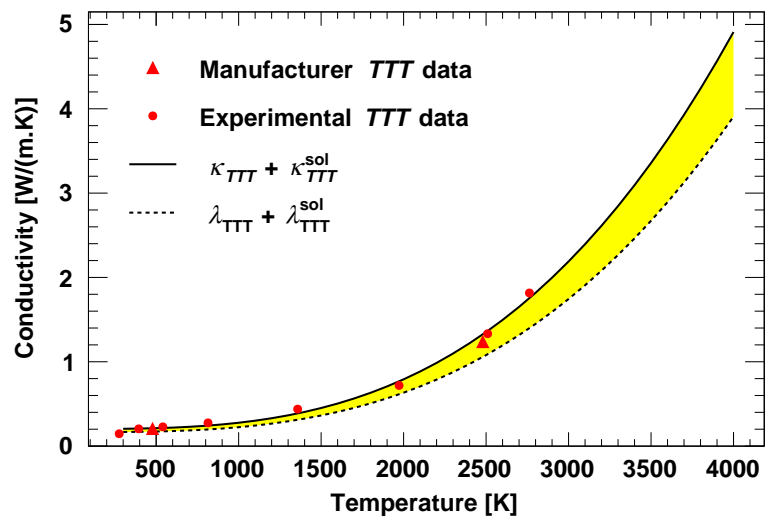

Figure 5: (Color online) Comparison of the total effective conductivity with experimental results presented by Tran et al. [8] and Fiber Materials Inc. [14]. The total effective conductivity includes the radiative conductivity calculated in this work, and the solid conductivity $\kappa_{T T T}^{\text {sol }}$ calculated by Panerai et al. [7]

conductivity value in the $T T T$ direction calculated by Panerai et al. [7], so that $\kappa_{T T T}^{\mathrm{eff}}=\kappa_{T T T}+\kappa_{T T T}^{\mathrm{sol}}$. To calculate $\kappa_{T T T}^{\text {sol }}$, they use a $1.0 \mathrm{~mm}^{3}$ cubic sample of FiberForm ${ }^{\circledR}$ in air, at $273.0 \mathrm{~K}$. Even though it is expected that the solid conductivity slightly decreases with an increase in temperature [15], a constant value of $\kappa_{T T T}^{\text {sol }}=0.203 \mathrm{~W} /(\mathrm{m} \cdot \mathrm{K})$ is used here. In addition, corresponding eigenvalue $\lambda_{T T T}^{\text {sol }}=0.165 \mathrm{~W} /(\mathrm{m} \cdot \mathrm{K})$ is calculated from the radiative conductivity tensor presented in [7], so that $\lambda_{T T T}^{\mathrm{eff}}=\lambda_{T T T}+\lambda_{T T T}^{\mathrm{sol}}$ is used for comparison.

The comparison is presented in Fig. 5. As can be seen, the manufacturer and experimental data, over the entire range of temperatures, are in very good agree- 


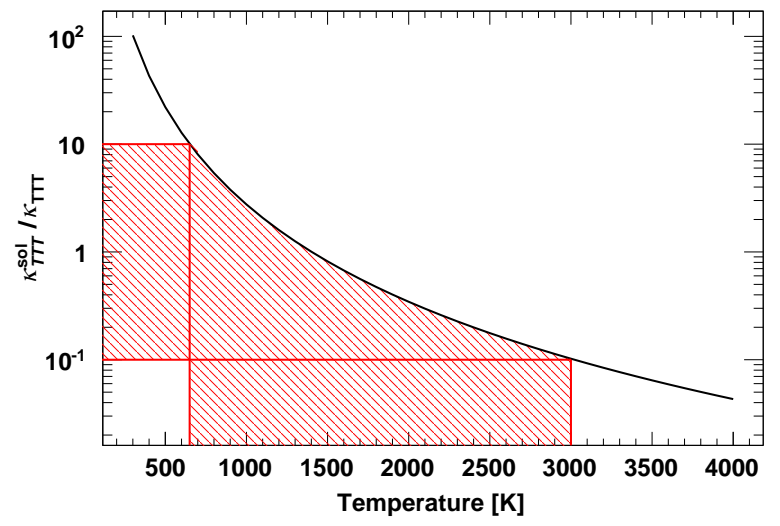

Figure 6: (Color online) Ratio of the solid and radiative conductivity as a function of temperature: the red region illustrates the temperature range where both terms are within $10 \%$ of each other, indicating that they both should be included in the effective conductivity calculation.

ment with the values bounded by the total effective conductivity $\kappa_{T T T}+\kappa_{T T T}^{\text {sol }}$ and corresponding principal axis total conductivity $\lambda_{T T T}+\lambda_{T T T}^{\text {sol }}$. Experimental data in the other directions are not readily available, but a factor of $\kappa_{I P} / \kappa_{T T T}=2.4$ is mentioned in Ref. [8], and a factor of 2.0 in Ref. [16]. Comparing to these ranges of values would also give a reasonable agreement for the two $\kappa_{I P}$, corresponding to the $\kappa_{x x}$ and $\kappa_{z z}$ values calculated here.

Figure 6 presents the $\kappa_{T T T}$ to $\kappa_{T T T}^{\text {sol }}$ ratio as a function of temperature, highlighting the respective contribution of the two terms. It can be seen that for temperatures lower than $650 \mathrm{~K}$, the radiative conductivity is likely negligible, accounting for only $10 \%$ of the effective conductivity. Likewise, at temperatures higher than $3000 \mathrm{~K}$, radiative effects are dominant and the solid conductivity can be neglected.

Finally, it is important to point out that the temperature-dependent gas phase conductivity and radiative absorption are not accounted for in the present work. Moreover, the geometry of the sample used for the radiative conductivity, $\kappa_{T T T}$, is different from the one used in Panerai et al. [7] to calculate $\kappa_{T T T}^{\mathrm{sol}}$.

\section{Conclusion}

The use of a X-ray microtomography generated geometry for computing radiative conductivity was demonstrated. A robust algorithm to calculate the geometric configuration factors among the planar surface elements of test geometry using triangular mesh generated by microtomography was constructed. The calculated values highlighted the heterogeneous behavior of fibrous material. The net radiation exchange model combined with Fourier's law was used to investigate the radiative energy flux distribution within the FiberForm ${ }^{\circledR}$ micro-structure. The developed model accounts for both the angular and thermal dependence of the radiative conductivity. The extracted values of the radiative conductivity tensor elements compare remarkably well with the experimental data, and manifest the anisotropic behavior of the material. The results also show that the cubic polynomial formulation of Rosseland model is valid for this case.

\section{Conflict of interest}

None declared.

\section{Acknowledgments}

The authors gratefully acknowledge the support of B. Plaster regarding the development of this work. Financial support for this work was provided by Kentucky EPSCoR and NASA RA Award NNX13AN04A and NASA Award NNX14AI97G. Part of this work was also performed under the Entry System Modeling Project (M.J. Wright program manager) of the NASA Game Changing Development (GCD) Program. Finally, the authors are also indebted to T. Cochell for pointing out the published experimental data, K. Bensassi and A. Munafo for providing helpful comments, and J.F. Wenk for a very helpful conversation on principal axis.

\section{References}

[1] A. van Eekelen, J. R. Lachaud, Numerical validation of an effective radiation heat transfer model for fiber preforms, Journal of Spacecraft and Rockets 48 (3) (2011) 534-537. doi: 10. 2514/1.51865.

[2] Y.-K. Chen, F. S. Milos, Ablation and thermal response program for spacecraft heatshield analysis, Journal of Spacecraft and Rockets 36 (3) (1999) 475-483. doi :10.2514/2.3469.

[3] R. A. S. Beck, D. M. Driver, M. J. Wright, H. H. Hwang, K. T. Edquist, S. A. Sepka, Development of the mars science laboratory heatshield thermal protection system, Journal of Spacecraft and Rockets 51 (4) (2014) 1139-1150. doi:10.2514/1. A32635.

[4] C. Szalai, E. Slimko, P. Hoffman, Mars science laboratory heatshield development, implementation, and lessons learned, Journal of Spacecraft and Rockets 51 (4) (2014) 1167-1173. doi: 10.2514/1. A32673.

[5] N. Nouri, A. Martin, Three dimensional radiative heat transfer model for the evaluation of the anisotropic effective conductivity of fibrous materials, International Journal of Heat and Mass Transfer 83 (2015) 629-635. doi:10.1016/j. ijheatmasstransfer.2014.12.041. 
[6] J. C. Ferguson, F. Panerai, S. C. C. Bailey, J. R. Lachaud, A. Martin, N. N. Mansour, Modeling the oxidation of lowdensity carbon fiber material based on micro-tomography, Carbon (2015) doi : 10.1016/j. carbon.2015.08.113.

[7] F. Panerai, J. C. Ferguson, J. R. Lachaud, A. Martin, M. J. Gasch, N. N. Mansour, Analysis of fibrous felts for flexible ablators using synchrotron hard x-ray micro-tomography, in: 8th European Symposium on Aerothermodynamics for Space Vehicles, Paper No. 89747, Lisbon, Portugal, 2015, pp. 1-8. doi:10.13140/RG.2.1.2661.0084.

[8] H. K. Tran, C. E. Johnson, D. J. Rasky, F. Hui, M. Hsu, T. Chen, Y. Chen, D. Paragas, L.Kobayashi, Phenolic impregnated carbon ablators (pica) as thermal protection systems for discovery missions, Technical Report NASA-TM-110440, NASA Ames Research Center, Moffett Field, CA (April 1997).

[9] B. N. Delaunay, Sur la sphère vide, in: International Congress of Mathematicians, Vol. 1, Toronto, ON, 1924, pp. 695-700.

[10] R. Siegel, J. R. Howell, M. P. Mengüç, Thermal radiation heat transfer, 5th Edition, CRC Press Taylor \& Francis, Boca Raton, FL, 2011.

[11] M. A. A. Mendes, V. Skibina, P. Talukdar, R. Wulf, U. Gross, D. Trimis, S. Ray, Experimental validation of simplified conduction-radiation models for evaluation of effective thermal conductivity of open-cell metal foams at high temperatures, International Journal of Heat and Mass Transfer 78 (2014) 112-120. doi:10.1016/j.ijheatmasstransfer. 2014.05 .058

[12] D. A. Codron, B. A. Cruden, T. S. Ho, Emission spectroscopy characterization of thermal protection system materials in archeated flows, in: 45th AIAA Plasmadynamics and Lasers Conference, AIAA Paper 2014-2112, 2014, pp. 1-12. doi:10. 2514/6.2014-2112.

[13] C. Garrod, J. Hurley, Symmetry relations for the conductivity tensor, Physical Review A 27 (3) (1983) 1487-1490, article 1487. doi:10.1103/PhysRevA.27.1487.

[14] Fiber Materials Inc. Fiberform $\AA$ rigid insulation [online] (2014). Retrieved from http://www.fibermaterialsinc.com/product/fiberform-rigidinsulation/ [cited 04/23/2015].

[15] Entegris, Inc., Properties and characteristics of graphite - for the semiconductor insdustry, Tech. Rep. 6205-7329CRE-0513, Entegris, Inc. (May 2013).

[16] H. Weng, A. Martin, Numerical investigation of thermal response using orthotropic charring ablative material, Journal of Thermophysics and Heat Transfer 29 (3) (2015) 429-438. doi : 10. 2514/1. T4576. 